\title{
Presentation and preliminary evaluation of the operational Early Warning System in Cyprus
}

\author{
K. Savvidou, S. Michaelides, K. A. Nicolaides, and P. Constantinides \\ Meteorological Service, Nicosia, Cyprus \\ Received: 6 March 2009 - Revised: 15 June 2009 - Accepted: 3 July 2009 - Published: 21 July 2009
}

\begin{abstract}
The Cyprus Early Warning System (EWS) and its validation are presented in this study. The EWS was developed within the framework of the Weather Risk Reduction in the Mediterranean project (RiskMed), the main objective of which is to warn the authorities and the public for severe weather phenomena, in order to minimise the impacts of weather related hazards. For the validation of the EWS, a comparison is made between the output of the system and the observations retrieved from 24 automatic weather stations operated by the Meteorological Service of Cyprus. From the validation, it resulted that the system underestimates the temperatures and overestimates the rain and the wind over Cyprus. These results can be attributed, firstly to the sea coverage of the study areas and secondly to the weakness of the weather model to represent topography. The EWS is a useful forecasting tool for local weather forecasters whose duties include the issue of warnings which are subsequently disseminated to the appropriate authorities acting for the safety of people and properties.
\end{abstract}

\section{Introduction}

The Weather Risk Reduction in the Mediterranean (RiskMed) is a European Union co-funded project in which Greece, Italy, Cyprus and Malta participated. The partners of this project are the University of Ioannina, Greece; the National Observatory of Athens, Greece; the Region of Epirus, Greece; the Italian National Research Council/Institute of Atmospheric Science and Climate, Italy; the Region of Calabria and Puglia, Italy; the University of Malta; and the Meteorological Service of Cyprus.

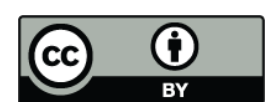

Correspondence to: K. Savvidou (lefele@cytanet.com.cy)
The RiskMed project can be characterized as a full-fledged system for weather forecasting; within the framework of this project an Early Warning System (EWS) was developed, which provides accurate and detailed weather forecasts of all meteorological hazards from extreme meteorological phenomena that happen in the central and east Mediterranean, such as heavy rain, thunderstorms, snowfall, strong surface winds, high waves and extremely low and high temperatures.

For the accomplishment of the above goal, the project combines satellite images, lightning data and meteo-marine sensors for the monitoring of severe weather events over the sea. Also, it uses high resolution meteorological and wave models for the provision of weather forecasts.

The EWS automatically detects, in accordance with specific thresholds, the areas where adverse weather phenomena are expected within the next two to three days and subsequently issues relevant warnings. These warnings are displayed in a user-friendly way, using pictograms (see Table 1) and colors (yellow, orange and red representing alert levels of increasing severity) on the web-page of RiskMed. Also, the warnings can be disseminated by electronic mail to the regional authorities. The Cyprus EWS is mainly a useful forecasting tool for local weather forecasters who make use of it in order to issue warnings which are subsequently disseminated to the appropriate authorities. For the more efficient utilization of the EWS outputs by the weather forecasters, a seven months validation of EWS outputs is performed, in order to quantitatively estimate errors. The validation is performed using ground weather stations across Cyprus.

The rest of the paper is organized as follows: Sect. 2 provides a short description of the RiskMed's components, as well as a description of the Cyprus EWS and the definition of the thresholds used. Section 3 provides the validation results. Finally, conclusions are drawn in Sect. 4. 
Table 1. The pictograms used in the EWS.

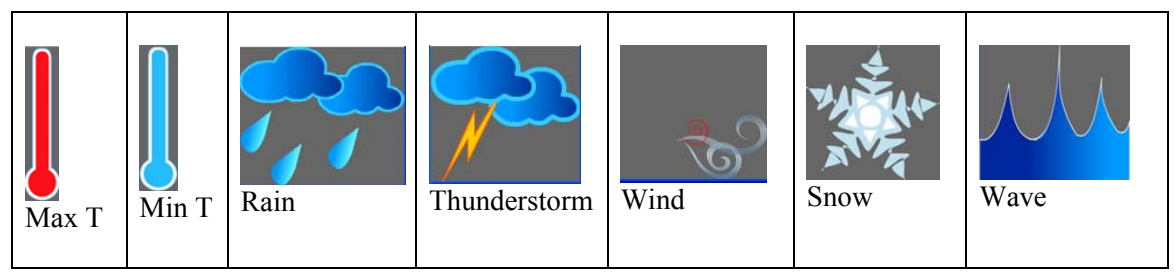

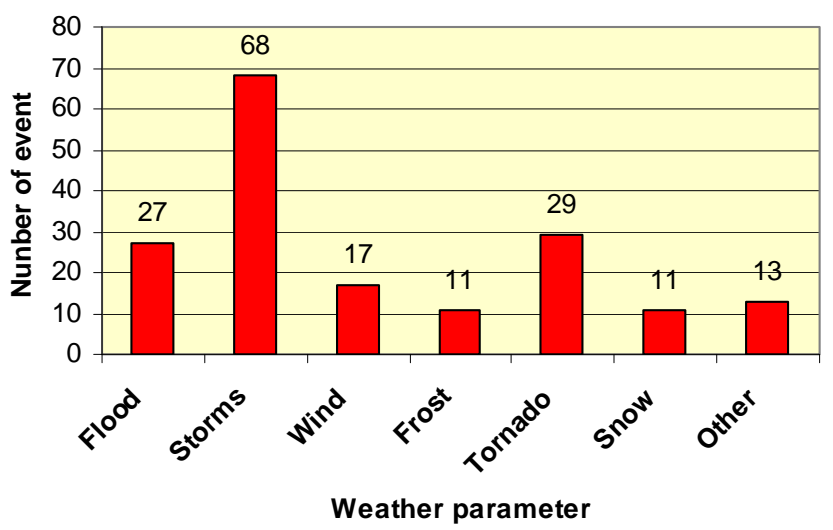

Fig. 1. Frequencies of weather phenomena for the period 1994 till 2004 resulting in hazardous events.

\section{RiskMed in Cyprus}

The RiskMed project has at its forefront an integrated weather forecasting tool. This integrated tool of the RiskMed EWS is made up of several components: lightning data provided by the ZEUS system (a lightning detection network operated by the National Observatory of Athens; for more details see Katsanos et al., 2007; Morales et al., 2007), satellite images, Cyprus latest observations and, finally, BOLAM (BOlogna Limited Area Model).

The BOLAM version used in the frame of this work is based on previous versions of the model described by Buzzi et al. (1994, 1997, 1998) and Buzzi and Foschini (2000). It is a hydrostatic model on an Arakawa $\mathrm{C}$ grid (rotated lat.-lon. coordinates) and uses the $\sigma$ vertical coordinate. BOLAM uses an explicit microphysical scheme with two water and three ice species (Schultz, 1995) as well as a convective parameterization scheme proposed by Kain and Fritsch (1993), with implementation of the modifications suggested by Spencer and Stensrud (1998), as it concerns the delay of downdrafts in newly developed convection.

For the operational implementation of BOLAM in the Cyprus Meteorological Service, two grids are used:

- The coarse grid with a 0.21 deg horizontal grid interval $(\sim 23 \mathrm{~km})$, covering the area of the Eastern Mediterranean.
- The fine grid with $0.07 \mathrm{deg}$ horizontal grid interval $(\sim 7 \mathrm{~km})$, covering the area of Cyprus and the adjacent seas.

In the vertical, 30 levels are used in the coarse grid and 40 levels in the fine grid, while model top has been set at about $10 \mathrm{hPa}$.

The initial and boundary data for the BOLAM model are provided by the operational global model GFS, at 6-h intervals. The duration of the forecasts is $72 \mathrm{~h}$, initialized every day using the 00:00 UTC GFS data.

The ZEUS ground network is a very low frequency (VLF) lightning detection network operated by the National Observatory of Athens (NOA). It consists of five European receivers.

\subsection{Description of the EWS}

During the period from 1994 to 2004, 176 cases of severe weather have been reported in Cyprus, resulting in damages to property, farming, as well as problems in the water and electricity supplies, transport, injuries of persons, and in some cases the loss of human life. Most of the damages are caused by storms, thunderstorms or hailstorms, secondly by tornados and thirdly by flood (Fig. 1). The EWS assists in better handling of the above problems by producing and disseminating in time relevant hazardous weather warnings.

The EWS automatically produces warnings for nine areas covering the island of Cyprus (Fig. 2), for specific ranges of values of five weather parameters and for three awareness levels.

These areas are: The Kormakitis area (1), Kyrenia area (2), Karpasia area (3), Pafos area (4), Troodos area (5), Mesaoria area (6), Ammochostos area (7), Limassol area (8) and the sea area Plateaux (9). It is obvious that in most areas the sea coverage is quite substantial and this will have a positive or negative contribution to the predicted variables and will, therefore, affect the issuance of warnings, accordingly. The parameters for which the system produces relevant warnings are the wind, temperature, rain and convective precipitation hereafter thunderstorm. For the above parameters, the thresholds for three levels of awareness i.e. yellow, orange and red were defined and are shown in Table 2. 
Table 2. Thresholds and awareness levels adopted by the Meteorological Service of Cyprus.

\begin{tabular}{llll}
\hline Parameter & Yellow level & Orange Level & Red Level \\
\hline Wind speed & $7-8$ Beaufort & $8-9$ Beaufort & $>9$ Beaufort \\
Rain & $5-2 \mathrm{~mm} / 6 \mathrm{~h}$ & $12-20 \mathrm{~mm} / 6 \mathrm{~h}$ & $>20 \mathrm{~mm} / 6 \mathrm{~h}$ \\
Thunderstorms & $5-12 \mathrm{~mm} / 6 \mathrm{~h}$ & $12-20 \mathrm{~mm} / 6 \mathrm{~h}$ & $>20 \mathrm{~mm} / 6 \mathrm{~h}$ \\
& Convective & Convective & Convective \\
& Precip. $>1 / 2$ & Precip. $>1 / 2$ & Precip. $>1 / 2$ \\
& Precip. & Precip. & Precip. \\
Maximum air temperature $\left(T_{\max },{ }^{\circ} \mathrm{C}\right)$ & $37<T_{\max } \leq 40$ & $40<T_{\max } \leq 43$ & $T_{\max }>43$ \\
Minimum air temperature $\left(T_{\min },{ }^{\circ} \mathrm{C}\right)$ & & & \\
For elevation $<400 \mathrm{~m}$ & $0<T_{\min } \leq 2$ & $-2<T_{\min } \leq 0$ & $T_{\min } \leq-2$ \\
\hline
\end{tabular}

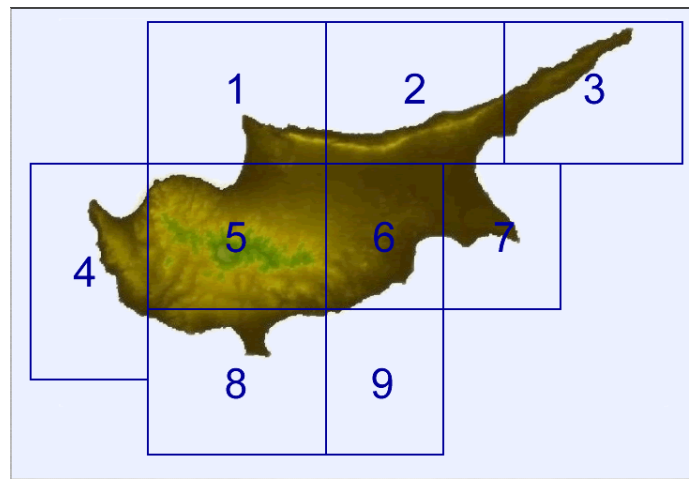

Fig. 2. The nine areas covering the island of Cyprus as used in the EWS.

The concept behind the EWS is simple. For every run of the BOLAM model a comparison is made between the values of the model output and the respective thresholds; if the former exceed the latter, the EWS produces warnings for the relevant parameters and areas (Fig. 3). The system has the ability to produce simultaneously warnings only for two meteorological parameters for each of the nine areas and for three awareness levels i.e. yellow, orange and red. These automatically produced warnings are monitored by the duty forecaster who subsequently proceeds with the issuance or not of the warnings which are disseminated to the various interested recipients, such as the civil aviation, the civil protection authorities, the authorities dealing with the sea areas of Cyprus, etc. The aforementioned authorities activate their own procedures for the better management of the expected situations.

\subsection{Definition of the thresholds}

Each country participating in RiskMed defines its own thresholds for which the EWS will produce warnings.

The levels of awareness and consequently the thresholds have been defined based on the repercussions that the meteo-

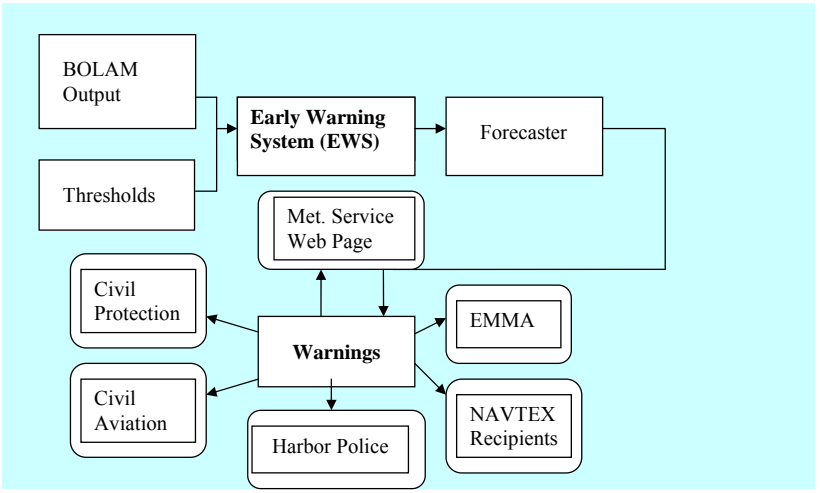

Fig. 3. Operational application of the EWS in Cyprus (EMMA is the early warning system of the European Meteorological Network of National Meteorological Services and NAVTEX - Navigational Telex - is an international direct-printing service for delivery of navigational and meteorological warnings and forecasts to ships.

rological phenomena have on the environment and on human life. The threshold definition is based on a fix value which is related to the frequency of appearance, while the level of awareness is directly related to the corresponding detrimental consequences and advice requirements.

For the determination of the thresholds, statistical data from a number of weather stations were used. For the wind, data in the period between 1985 and 2005 for the weather stations of Athalassa, Pafos, Larnaka and Agros were studied. The values of the wind with frequency of appearance between 0.1 and 0.2 times per year correspond to the red awareness level, between 0.2 and 0.4 times per year correspond to the orange awareness level and between 0.4 and 1 times per year correspond to the yellow awareness level.

For temperature, data in the period between 1991 and 2005 and for the stations of Athalassa, Larnaka and Pafos were used. For the maximum temperature, values with frequency of appearance up to 0.3 times per year correspond to the red awareness level, values with frequency between 0.3 and 6 times per year correspond to the orange awareness level and finally values with frequency between 6 and 21 times per 
Table 3. Number of warnings for wind, rain and thunderstorm that the EWS issued automatically in the period between 20/7/2007-29/2/2008.

\begin{tabular}{lrrrrrrrrr}
\hline $\begin{array}{l}\text { Nr of } \\
\text { WNGS }\end{array}$ & \multicolumn{1}{c}{ Wind } & \multicolumn{3}{c}{ Rain } & \multicolumn{5}{c}{ Thunderstorms } \\
\hline Area & Yellow & Orange & Red & Yellow & Orange & Red & Yellow & Orange & Red \\
\hline 1 & 129 & 22 & 0 & 7 & 5 & 4 & 139 & 35 & 10 \\
2 & 120 & 18 & 1 & 5 & 4 & 4 & 99 & 24 & 8 \\
3 & 85 & 11 & 1 & 5 & 5 & 7 & 85 & 23 & 5 \\
4 & 53 & 12 & 0 & 7 & 1 & 3 & 100 & 22 & 3 \\
5 & 25 & 1 & 0 & 11 & 6 & 5 & 119 & 23 & 5 \\
6 & 50 & 9 & 0 & 9 & 2 & 1 & 54 & 10 & 2 \\
7 & 41 & 9 & 2 & 6 & 2 & 4 & 68 & 9 & 2 \\
8 & 76 & 7 & 1 & 4 & 7 & 2 & 83 & 12 & 1 \\
9 & 64 & 12 & 1 & 11 & 4 & 2 & 43 & 3 & 0 \\
\hline
\end{tabular}

Table 4. Number of warnings for $T_{\max }$ and $T_{\min }$ that the EWS issued automatically in the period between 20/7/2007-29/2/2008.

\begin{tabular}{lrrrrrr}
\hline $\begin{array}{l}\text { Nr of } \\
\text { WNGS }\end{array}$ & \multicolumn{7}{c}{$T_{\max }$} & \multicolumn{7}{c}{$T_{\min }$} \\
\hline Area & Yellow & Orange & Red & Yellow & Orange & Red \\
\hline 1 & 30 & 6 & 0 & 26 & 4 & 0 \\
2 & 31 & 7 & 0 & 5 & 0 & 0 \\
3 & 1 & 0 & 0 & 0 & 0 & 0 \\
4 & 1 & 0 & 0 & 0 & 0 & 0 \\
5 & 20 & 2 & 0 & 15 & 0 & 0 \\
6 & 19 & 2 & 0 & 4 & 0 & 0 \\
7 & 1 & 0 & 0 & 0 & 0 & 0 \\
8 & 0 & 0 & 0 & 0 & 0 & 0 \\
9 & 0 & 0 & 0 & 0 & 0 & 0 \\
\hline
\end{tabular}

year correspond to the yellow awareness level.

For the rain, data from 14 raingauges and in the period between 1991 and 2005 were used. The corresponding frequencies of appearance per year are: equal or less than 0.1 corresponds to the red awareness level, between 0.1 and 0.3 to the orange awareness level and between 0.3 and 5 to the yellow awareness level. For thunderstorm, the same criteria as those for rain are adopted but under the assumption that rain is accompanied by thunder.

The three levels of awareness are defined as follows:

- Yellow: The weather is potentially dangerous. The weather phenomena that have been forecast are not unusual but may be affect outdoor activities.

- Orange: The weather is dangerous. Unusual meteorological phenomena have been forecast. Damage and casualties are likely to happen. Be very vigilant and keep regularly informed about the detailed expected meteorological conditions.

- Red: The weather is very dangerous. Exceptionally intense meteorological phenomena have been forecast. Major damage and accidents are likely, in many cases with threat to life and limb, over a wide area. Keep frequently informed about detailed expected meteorological conditions and risks.

The results from the above statistics define the Cyprus' thresholds which are shown in Table 2.

In Tables 3 and 4 show the number of warnings that the EWS issued for each variable for the period between 20/7/2007 till the 29/2/2008 is shown. The number of warnings of maximum and minimum temperature is low and in some of the sub-areas is even zero; this might indicate that a re-definition of the temperature thresholds might be needed. A reconsideration of the rain red threshold is needed because the percentage of the red warnings is quite high compared to the percentages of the yellow and orange warnings. Overall, it is considered that a re-definition of thresholds for each area and each parameter will give better results.

\section{Validation of the EWS}

In order to validate the EWS, a comparison is made between the output of the system and the observations retrieved from 24 automatic weather stations operated by the Meteorological Service of Cyprus (Fig. 4) for the period from 20/7/2007 till 29/2/2008. The Probability Of Detection (POD) and the False Alarm Rate (FAR) for each parameter and for each area were calculated. The validation is made only for the areas 4 , 5, 6, 7 and 8 of Fig. 2. There is no accessibility to measurements from areas 1,2 and 3. Also, area 9 is wholly maritime, without any data available for validation. In the following, the validation is briefly discussed and presented for each of the meteorological variables handled by the system. 


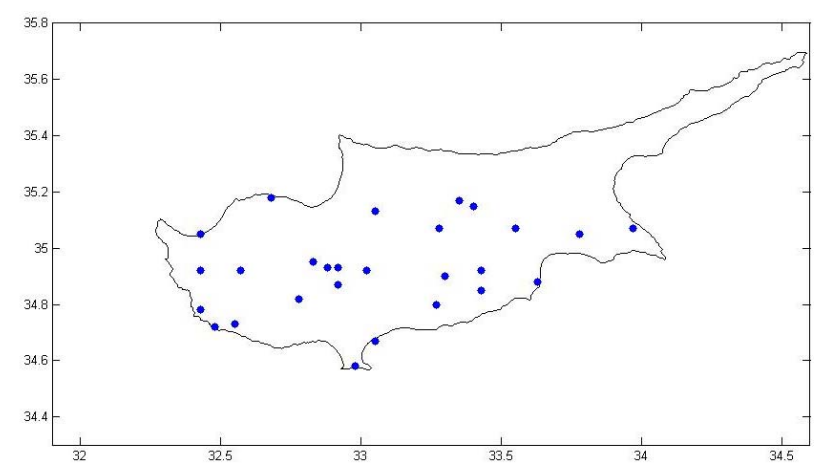

Fig. 4. Distribution of the surface stations used in the validation of the EWS.

Table 5. POD and FAR for $T_{\max }$.

\begin{tabular}{lllllll}
\hline $\begin{array}{l}\text { Awareness } \\
\text { Level }\end{array}$ & Yellow & \multicolumn{3}{l}{ Orange } & \multicolumn{2}{l}{ Red } \\
\hline Area & POD & FAR & POD & FAR & POD & FAR \\
\hline 4 & 20 & 0 & 0 & - & - & - \\
5 & 44 & 8 & 15 & 0 & 0 & - \\
6 & 34 & 0 & 22 & 0 & 0 & - \\
7 & 33 & 33 & 50 & 0 & - & - \\
8 & 0 & - & - & - & - & - \\
\hline
\end{tabular}

\subsection{Temperature}

\subsubsection{Maximum temperature $\left(T_{\max }\right)$}

The results for POD and FAR for $T_{\max }$ and for each area are shown in Table 5. In areas 4, 7 and 8, the percentage of land coverage is small; consequently, the temperature of these areas is largely affected by the sea temperature and for this reason quite few cases of extreme high temperature were reported (Fig. 5). Cases of extreme high temperature were reported mainly over areas 5 and 6 . The skill of the EWS to detect them is rather low, while almost all the forecast cases were confirmed by observations. Also, no red level warning was produced, while three cases for area 5 and two cases for area 6 exceeding the respective threshold were recorded. This leads to the conclusion that the maximum temperature is underestimated by the BOLAM model.

\subsubsection{Minimum temperature $\left(T_{\min }\right)$}

The results for POD and FAR for the $T_{\min }$ temperature and for each area are shown in Table 6. The sea coverage has the same influence as for the extreme high temperature and on the extreme low temperature of areas 4 and especially 7 . Quite a lot events were reported in area 7 but not forecast (Fig. 6). The POD for the areas 5 and 6 is very low, while the

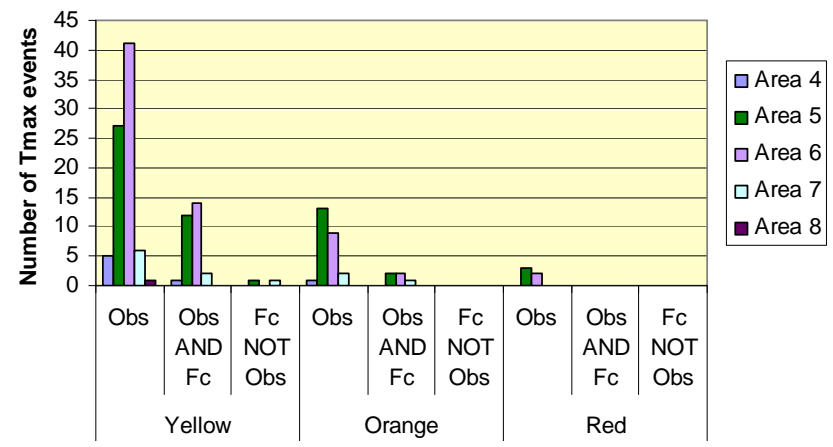

Fig. 5. Number of $T_{\max }$ events: Observed (Obs), Observed and Forecast (Obs AND Fc) and Forecast but not Observed (Fc NOT Obs), for various areas and the three awareness levels.

Table 6. POD and FAR for $T_{\min }$.

\begin{tabular}{lllllll}
\hline $\begin{array}{l}\text { Awareness } \\
\text { Level }\end{array}$ & Yellow & & \multicolumn{2}{l}{ Orange } & \multicolumn{2}{l}{ Red } \\
\hline Area & POD & FAR & POD & FAR & POD & FAR \\
\hline 4 & 0 & - & - & - & - & - \\
5 & 22 & 60 & 0 & - & - & - \\
6 & 12 & 33 & 0 & - & - & - \\
7 & 0 & - & 0 & - & 0 & - \\
8 & - & - & - & - & - & - \\
\hline
\end{tabular}

FAR in very high for the yellow awareness level. For orange and red awareness level the POD is zero. The conclusion is that the minimum temperature is underestimated by the BOLAM model.

The BOLAM output for the surface temperature refers to the temperature at $2 \mathrm{~m}$, thus the night surface cooling and the day surface heating are partly considered by the model; this can be a factor justifying the generally weak skill of the system in forecasting temperature.

From Tables 5 and 6, the POD values of the minimum temperature are much lower than the values of the maximum ones. This can be attributed to the weaker skill of the BOLAM model in predicting minimum temperatures than the maximum ones. According to Savvidou et al. (2009), the standard deviation of the mean error of the temperature during night is higher than the one during day.

\subsection{Wind}

Although the skill of the BOLAM model in predicting the wind is quite good; especially over inland areas (Savvidou et al., 2009), the evaluation resulted in quite high values for FAR for most of the areas (Table 7). This can be attributed to the high sea coverage of the areas, where the effect of frictional retardation is less than over land. Consequently, 


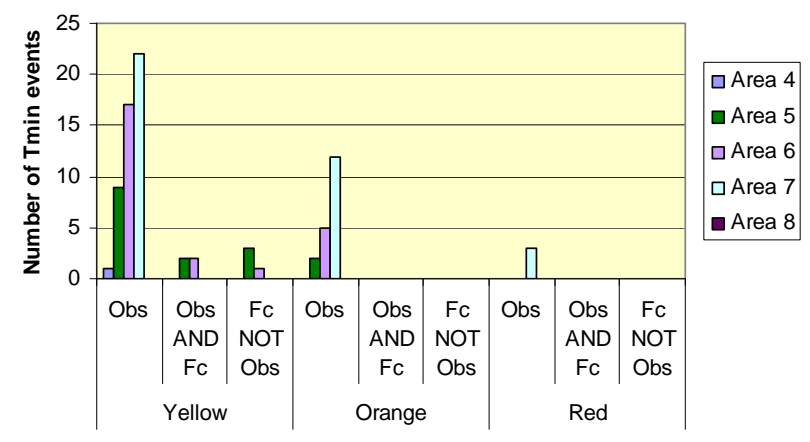

Fig. 6. Same as Fig. 5 but for $T_{\min }$.

Table 7. POD and FAR for the wind.

\begin{tabular}{lllllll}
\hline $\begin{array}{l}\text { Awareness } \\
\text { Level }\end{array}$ & Yellow & \multicolumn{3}{l}{ Orange } & Red \\
\hline Area & POD & FAR & POD & FAR & POD & FAR \\
\hline 4 & 100 & 72 & 100 & 90 & - & - \\
5 & 39 & 13 & 28 & 38 & - & - \\
6 & 48 & 58 & 100 & 63 & - & - \\
7 & 50 & 84 & 100 & 90 & - & - \\
8 & 57 & 79 & 50 & 88 & - & - \\
\hline
\end{tabular}

these wind events were not observed by the observer at the land stations and were considered as false alarm events.

It can be inferred that the wind is overestimated by the EWS for both the lower awareness levels, namely, yellow and orange, almost in all areas, with the lowest overestimation over area 5 which is a mountainous. For the red awareness level, only 2 cases were observed all over Cyprus which were not forecast; three cases were forecast all over Cyprus which were not confirmed through observation (Fig. 7).

\subsection{Rain}

The evaluation results for the rain events were very poor (low values for POD, high values for FAR) (Table 8) The low values of POD for all awareness levels are probably due to the weakness of the BOLAM model to represent correct the local topography. The high values of FAR can be attributed partly to the fact that the sea coverage of the areas 4,7 and 8 is large and some events which happened over the sea were not observed by the observer at the land stations; nevertheless, these events are considered as false alarm events. For the red awareness level, one event was observed in each area except in area 5 in which 9 events were observed two of which were predicted by the model (Fig. 8).

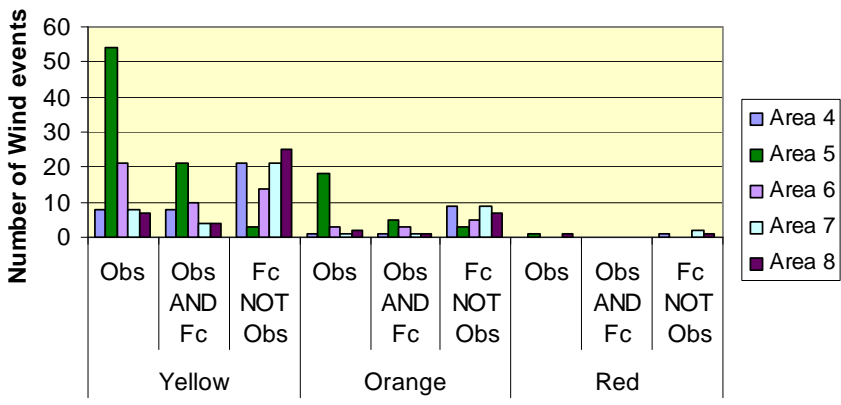

Fig. 7. Same as Fig. 5 but for Wind.

Table 8. POD and FAR for rain.

\begin{tabular}{lllllll}
\hline $\begin{array}{l}\text { Awareness } \\
\text { Level }\end{array}$ & Yellow & & Orange & Red \\
\hline Area & POD & FAR & POD & FAR & POD & FAR \\
\hline 4 & 8 & 60 & 0 & 100 & 0 & 100 \\
5 & 8 & 67 & 25 & 33 & 22 & 50 \\
6 & 11 & 83 & 17 & 50 & 0 & 100 \\
7 & 0 & 100 & 50 & 50 & 0 & 100 \\
8 & 17 & 75 & 0 & 100 & 0 & 100 \\
\hline
\end{tabular}

\subsection{Thunderstorm}

The validation for the thunderstorms is performed only for the areas 4,8 and 6 with the use of the reports registered by trained professional observers at Pafos Airport, Akrotiri, Larnaka Airport and at Athalassa weather stations (see Table 9).

The POD for the yellow and orange awareness levels is rather good, especially for areas 4 and 6, but the FAR is high; this leads to the conclusion that either the thunderstorms are overestimated by the BOLAM model (Fig. 9) or, due to high sea coverage of the areas 4 and 8 , some events can happen over the sea but not being observed by the land station. These events are considered as false alarm events. For the red awareness level, one event per area was observed which was not forecast, while a couple of events were forecast but not observed.

\section{Concluding remarks}

The EWS was developed and deployed in order to provide accurate and detailed weather forecasts and to disseminate the corresponding warnings of meteorological hazards due to extreme meteorological phenomena that happen in the area of Cyprus, for better awareness of the public and the local authorities. The presentation of the EWS is simple and easy to understand by everyone, by using a color code and pictograms (see the official web site of RiskMed at www.riskmet.net). 


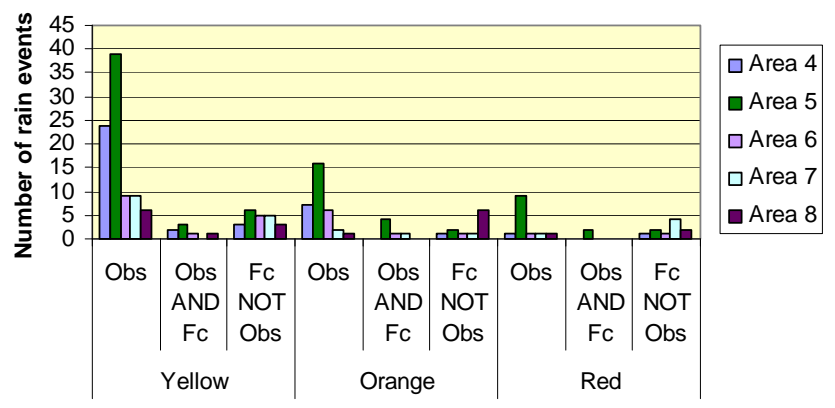

Fig. 8. Same as Fig. 5 but for Rain.

Table 9. POD and FAR for thunderstorms.

\begin{tabular}{lllllll}
\hline $\begin{array}{l}\text { Awareness } \\
\text { Level }\end{array}$ & Yellow & \multicolumn{2}{l}{ Orange } & Red \\
\hline Area & POD & FAR & POD & FAR & POD & FAR \\
\hline 4 & 100 & 84 & 50 & 88 & 0 & 100 \\
6 & 80 & 86 & 75 & 57 & 0 & 100 \\
8 & 50 & 94 & N.A & 100 & 0 & 100 \\
\hline
\end{tabular}

A preliminary validation of the EWS was performed in this study by comparing the output of the system with the observations retrieved from 24 automatic weather stations. Based on the construction of a contingency table, the probability of detection of each weather parameter and the false alarm rate for only of five areas were calculated.

The validation results show that the best combination of POD and FAR was found for the maximum temperature while the worst, for the rain. Quite good values of POD were found for the wind and thunderstorm but the respective values of FAR were very high.

Overall the system underestimates the temperature and overestimates the wind, rain and thunderstorms. These results are due firstly to the limitation of the BOLAM to represent the local topography, the day heating and the night cooling, secondly to the high sea coverage of most of the areas and thirdly to the small period of validation.

It is obvious that the sea coverage of the areas is a disadvantage in the evaluation procedure, resulting in high values of FAR. In order to minimize this disadvantage, observations obtained from radar and satellite will also be used in a future study.

Acknowledgements. This study was undertaken within the framework of project RiskMed which was co-funded by the European Union (Interreg IIIB) and the Government of Cyprus.

Edited by: A. Bartzokas

Reviewed by: two anonymous referees

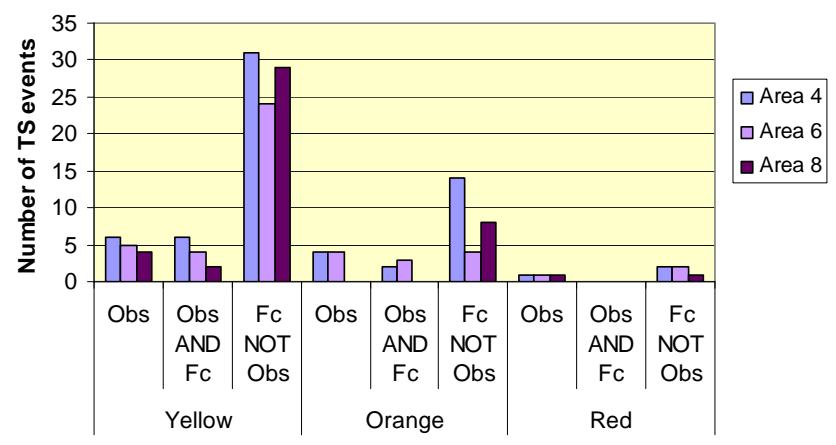

Fig. 9. Same as Fig. 5 but for Thunderstorms.

\section{References}

Buzzi, A., Fantini, M., Malguzzi, P., and Nerozzi, F.: Validation of a limited area model in cases of Mediterranean cyclogenesis: surface fields and precipitation scores, Meteorol. Atmos. Phys., 53, 137-153, 1994.

Buzzi, A., Cadelli, R., and Malguzzi, P.: Low level jet simulation over the Antarctic ocean, Tellus, 49A, 263-276, 1997.

Buzzi A., Tartaglione, N., and Malguzzi, P.: Numerical simulations of the 1994 Piedmont flood: role of orography and moist processes, Mon. Weather Rev., 126, 2369-2383, 1998.

Buzzi, A. and Foschini, L.: Mesoscale meteorological features associated with heavy precipitation in the southern Alpine region, Meteorol. Atmos. Phys., 72, 131-146, 2000.

Kain, J. S. and Fritsch, J. M.: Convective parameterization for mesoscale models: The Kain-Fritsch scheme, The Representation of Cumulus in numerical models, American Meteorological Society, No 46, Meteor. Mon., 165-177, 1993.

Katsanos, D., Lagouvardos, K., Kotroni, V., and Argiriou, A.: Combined analysis of rainfall and lightning data produced by mesoscale systems in the Central and Eastern Mediterranean, Atmos. Res., 83, 55-63, 2007.

Morales, C. A., Anagnostou, E. N., Williams, E., and Kriz, J. S.: Evaluation of peak current Polarity Retrieved by the ZEUS LongRange Lightning Monitoring System, IEEE Geosci. Remote S., 4, 32-36, 2007.

Savvidou K., Lagouvardos K., Constantinides P., Kotroni V., and Michaelides S.: Verification of the BOLAM model over the area of Cyprus, Advances in Geosciences, submitted for publication, 2009.

Schultz, P.: An explicit cloud physics parameterization for operational numerical weather prediction, Mon. Weather Rev., 123, 3331-3343, 1995.

Spencer, P. L. and Stensrud, D. J.: Simulating flash flood events: Importance of the subgrid representation of convection, Mon. Weather Rev. 126, 2884-2912, 1998. 